\title{
Belgeo
}

Revue belge de géographie

\section{Editorial : Dépasser la crise en Afrique}

Stéphane Bastin et Jean-Michel Decroly

\section{(2) OpenEdition}

Journals

Édition électronique

URL : http://journals.openedition.org/belgeo/10090

DOI : 10.4000/belgeo.10090

ISSN : 2294-9135

Éditeur :

National Committee of Geography of Belgium, Société Royale Belge de Géographie

Édition imprimée

Date de publication : 31 décembre 2007

Pagination : 399-402

ISSN : 1377-2368

\section{Référence électronique}

Stéphane Bastin et Jean-Michel Decroly, «Editorial : Dépasser la crise en Afrique », Belgeo [En ligne], 4 | 2007, mis en ligne le 18 octobre 2013, consulté le 22 septembre 2020. URL : http:// journals.openedition.org/belgeo/10090 ; DOI : https://doi.org/10.4000/belgeo.10090

Ce document a été généré automatiquement le 22 septembre 2020.

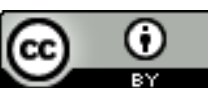

Belgeo est mis à disposition selon les termes de la licence Creative Commons Attribution 4.0 International. 


\title{
Editorial : Dépasser la crise en Afrique
}

\author{
Stéphane Bastin et Jean-Michel Decroly
}

La majeure partie de l'Afrique sub-saharienne est aujourd'hui confrontée à une indéniable situation de crise. Avec la chute du prix des matières premières sur les marchés mondiaux à partir de la fin des années 1970 s'enraye le modèle de l'économie de rente - qui fonctionnait essentiellement par prélèvements sur les produits agricoles et miniers ainsi que sur l'aide extérieure. L'amenuisement subséquent d'une importante source de revenus pour les états du sous-continent fait craindre leur manque de solvabilité aux financiers privés occidentaux qui, après y avoir largement soutenu les investissements publics, s'en détournent dès le début des années 1980. Ceci précipite leur banqueroute et, pris dans l'engrenage de la dette, ils sont contraints pour accéder à de nouveaux financements - de se plier aux exigences des institutions financières internationales (Banque Mondiale et FMI), d'autant que l'aide publique au développement s'effondre à son tour suite à la chute du mur de Berlin et à la fin de la guerre froide. En contrepartie des prêts consentis par ces institutions, les états africains se voient donc imposer une série de mesures d'ajustement structurel politiques d'austérité, dérégulation et libéralisation des prix - ayant pour effet d'accélérer l'érosion de leur pouvoir interventionniste. Les caisses de crédit rural disparaissent, les entreprises étatiques sont dissoutes, les infrastructures routières se dégradent, les aménagements hydrauliques ne sont plus entretenus... Inscrite dans un contexte de forte croissance démographique ( $+2,8 \%$ en moyenne par an depuis 1980$)$ et d'insécurité climatique persistante, cette crise des complexes politico-économiques a des effets catastrophiques sur la région, du moins à l'aune des statistiques économiques, alimentaires et sanitaires classiquement utilisées. Et si le retour des rentes stratégiques depuis le début des années 2000, parallèlement au redéploiement des intérêts américains et à la croissance économique de la Chine, n'a pas encore eu d'impact tangible sur l'amélioration globale de telles statistiques, il a, quoi qu'il en soit, déjà fait sentir ses effets sur l'exacerbation des tensions régionales (conflit du Darfour, rébellion touarègue....). 
Cette crise structurelle n'est pas sans alimenter - de la part de certains médias ou avec leur complaisance - un discours fortement teinté d'“afro-pessimisme", attribuant la "faillite" du sous-continent à des populations incapables de réaction autonome. D'après Georges Courade (2006), analysant les traits les plus saillants des idées reçues sur l'Afrique, il est possible de classer les thèses afro-pessimistes selon qu'elles imputent la crise à un héritage de l'oppression, à une aliénation culturelle ou aux mentalités des Africains eux-mêmes. C'est ce dernier argument qui est privilégié par Stephen Smith dans sa Négrologie (2003), pour laquelle la situation actuelle de l'Afrique incomberait à des populations "se refusant à entrer dans la modernité autrement qu'en passager clandestin ou en consommateur vivant aux crochets du reste du monde" (Smith, 2003, p. 230, cité par Bastin et Fromageot). Dans leur récente publication tirée du film We feed the world, Erwin Wagenhofer et Max Annas estiment quant à eux que l'unique alternative s'ouvrant au paysan sénégalais dont la production de tomates est concurrencée par celle importée d'Andalousie est "s'il en a encore l'énergie [d'] émigrer, au péril de sa vie, en passant par le Détroit de Gibraltar" (Wagenhofer et Annas, 2007, p. 34). Attendre résignés une aide hypothétique ou, à défaut, tenter de s'embarquer pour l'Europe... ce sont là les rares options qui s'offriraient encore aux habitants d'un enfer terrestre que les manifestations de crise - pauvreté, corruption, conflits, épidémies, désertification ou famines - seraient désormais seules à façonner.

3 Cette représentation d'une Afrique agonisante est pourtant loin de correspondre à la réalité. Celle-ci se révèle en effet beaucoup plus complexe lorsque l'observation de terrain fait émerger le foisonnement de dynamiques culturelles et économiques de dépassement de la crise, attestant de réelles capacités de développement endogène. $\mathrm{A}$ partir de recherches empiriques conduites en territoire ouest-africain, c'est aux formes spatiales originales produites par de telles dynamiques que sont consacrées les contributions présentées dans ce numéro.

4 Sébastien Bainville et Marc Dufumier montrent ainsi comment le transfert de techniques (équipements attelés surtout) acquises dans le cadre de la promotion étatique du coton vers la céréaliculture pluviale permet aux paysans du Sud-Mali de passer de l'abattis-brûlis à une culture en continu sans diminution des rendements. Et si les auteurs avancent que ce changement est de surcroit favorable à une amélioration de la rémunération du travail, ils se gardent néanmoins de verser dans un afrooptimisme excessif - observant qu'il est parfois freiné par une recrudescence de la scission des familles élargies.

5 C'est d'intensification des pratiques agricoles dont il est également question dans la publication proposée par Stéphane Bastin et Audrey Fromageot, pour qui la récente propagation du maraîchage dans les campagnes songhay et sénoufo doit avant tout être interprétée comme le résultat d'une capacité des exploitants à puiser dans leurs héritages techniques lorsque, à partir de la fin des années 1980, il leur a fallu diversifier leurs sources de revenus pour faire face aux défaillances enregistrées par leur système d'activité respectif.

6 Enfin, au-delà du fait que se confirme à la lecture d'Anneleen Vanderveken le pouvoir d'attraction actuel de certaines villes sahéliennes (Niamey en l'occurrence), jadis plutôt délaissées par l'exode temporaire de saison sèche au profit des grandes villes du Golfe de Guinée, l'auteure montre comment les fondeurs allochtones implantés dans la capitale nigérienne sont arrivés à y garantir leur maintien par le jeu de réadaptations langagières. 


\section{BIBLIOGRAPHIE}

CHRETIEN J.-P. et al. (2005), “Misères de l'afro-pessimisme”, Afrique et Histoire, 3, pp. 183-211.

COURADE G. (dir.) (2006), L'Afrique des idées reçues, Coll. Mappemonde, Belin.

DUBRESSON A. \& RAISON J.-P. (2003), L'Afrique subsaharienne. Une géographie du changement, Paris, Armand Colin.

GOSSELAIN O. \& BASTIN S. (2005), "Famine, routine et journalisme”, Hémisphères, 31, pp. 6-7.

RAISON J.-P. (1993), "Les formes spatiales de l'incertitude en Afrique contemporaine”, Travaux de l'Institut de Géographie de Reims, numéro spécial Espaces africains en crise : formes d'adaptation et de réorganisation, 83-84, pp. 5-18.

SMITH S. (2003), Négrologie. Pourquoi l'Afrique meurt, Calmann-Lévy.

VANDERMOTTEN C. \& MARISSAL P. (2003), La production des espaces économiques. Tome 2, Editions de l'Université de Bruxelles.

WAGENHOFER E. \& ANNAS M. (2007), Le marché de la faim. Le livre du film We feed the world, Actes Sud.

\section{AUTEURS}

\section{STÉPHANE BASTIN}

IGEAT - ULB, sbastin@ulb.ac.be

JEAN-MICHEL DECROLY

IGEAT - ULB, jmdecrol@ulb.ac.be 